\title{
STUDY OF UNEVEN FILLS TO CURE THE COUPLED-BUNCH INSTABILITY IN SRRC
}

\author{
M. H. Wang*, P. J. Chou, SRRC, Hsinchu, 300, Taiwan \\ A. W. Chao, SLAC, Stanford, CA 94309, USA
}

\begin{abstract}
The performance of the $1.5-\mathrm{GeV}$ storage ring light source TLS in SRRC has been limited by a longitudinal coupled-bunch beam instability. To improve the performance of the TLS, the beam instability has to be suppressed. One possible way considered for the TLS to suppress its coupled-bunch instability uses uneven filling patterns according to the theory of Prabhakar[1]. By knowing the harmful high-order-modes (HOMs), a special filling pattern can be designed to utilize either mode coupling or Landau damping to cure beam instability. In TLS the HOMs are contributed from the Doris RF cavity installed in the storage ring. The HOMs of a 3-D Doris cavity was numerically analyzed. Filling patterns with equal bunch current according to theory had been calculated to cure the most harmful HOM. A longitudinal particle tracking program was used to simulate the coupled-bunch beam instability with both the uniform filling and the special designed filling. Filling pattern with unequal bunch current was also studied. The results of the simulation were discussed and compared to the theory.
\end{abstract}

\section{INTRODUCTION}

The uniform-filled coupled-bunch beam instability has been well known. Consider a stored beam that consists of $\mathrm{M}$ identical macroparticle bunches of charge q, uniformly filled in M RF buckets, with a constant interbunch spacing $T_{b}=T_{0} / M$. Where $T_{0}$ is revolution period of the beam. The effect of the wake field on the longitudinal arrival time of $n^{\text {th }}$ bunch relative to the synchronous bunch can be described as:

$$
\ddot{\tau}_{n}+\frac{2}{\tau_{\text {rad }}} \dot{\tau}_{n}+\omega_{s}^{2} \tau_{n}=-\frac{\eta e}{E_{0} T_{0}} V_{n}^{w k}(t) .
$$

Where $\eta$ is the slip factor, $E_{0}$ is the energy of the synchronous particle, $\tau_{\text {rad }}$ is the radiation damping time, $\omega_{s}$ is the angular synchrotron frequency, and $V_{n}^{w k}(t)$ is the wake voltage due to the superposition of wake forces of all preceding macroparticles. If the wavelengths of the relevant wake fields are much larger than the bunch length the wake of a bunch is approximated by that of a point charge q,

$V_{n}^{w k}(t)=q \sum_{p=-\infty}^{\infty} \sum_{k=0}^{M-1} W\left[t_{n, k}^{p}+\tau_{n}(t)-\tau_{k}\left(t-t_{n, k}^{p}\right)\right]$,

where $\tau_{k}\left(t-t_{n, k}^{p}\right)=(p M+n-k) T_{b}$ is the bunch spacing between the $n^{t h}$ bunch at present and the $k^{t h}$ bunch $\mathrm{p}$ turns

*mhwang@srrc.gov.tw ago. In this system there exists $M$ eigenmodes with $M$ set of eigen vectors

$$
\begin{array}{r}
v_{\ell}=\left[1 e^{j \ell \theta} e^{2 j \ell \theta} \ldots \ldots e^{(M-1) j \ell \theta}\right]^{T} / \sqrt{M}, \\
\ell=0,1, \ldots, M-1, \quad \theta=2 \pi / M .
\end{array}
$$

This forms the set we call Even Fill Eigen Mode(EFEM). If the bunches are oscillating in mode $\ell, \tau_{n} \propto e^{j \Omega^{\ell} t}$, the eigen mode frequency $\Omega^{\ell}$ is given by:

$$
j \Omega^{\ell}+\left(\frac{1}{\tau_{\text {rad }}}-j \omega_{s}\right)=\lambda_{l},
$$

with

$$
\begin{aligned}
& \lambda_{l}=\frac{\alpha e f_{r f} I_{0}}{2 E_{0} \nu_{s}}\left[Z^{\| e f f}\left(l \omega_{0}+\omega_{s}\right)-Z^{\| e f f}(0)\right], \\
& Z^{\| \operatorname{eff}}(\omega)=\frac{1}{\omega_{r f}} \sum_{p=-\infty}^{\infty}\left(p M \omega_{0}+\omega\right) Z^{\|}\left(p M \omega_{0}+\omega\right),
\end{aligned}
$$

$Z^{\|}(\omega)=\int_{-\infty}^{\infty} W(t) e^{-j \omega t} d t$ is the impedance. The second term in Eq. (5) contributes an incoherent synchrotron tune shift and the first descibes the instability.

In real operation the accelerator is usually running in an uneven filling pattern. The uneven-fill complicats the analysis of coupled-bunch beam instability. However from the study of Dr. Prabhakar[1] the uneven filling pattern can be utilized to couple the damp and antidamp modes to suppress the beam instability. In this report we study the filling pattern to cure the most harmful HOM from rf cavity at TLS.

\section{UNEVEN FILL}

For uneven filling the equation of the longitudinal oscillation is the familiar Eq. (1), except now the wake voltage in Eq. (2) is replaced by:

$V_{n}(t)=\sum_{p=-\infty}^{\infty} \sum_{k=0}^{M-1} q_{k} W\left[t_{n, k}^{p}+\tau_{n}(t)-\tau_{k}\left(t-t_{n, k}^{p}\right)\right]$.

The charge is different for different bunches. This makes the solution of all bunches no longer form an orthogonal basis. However one can use the Even Fill Eigen Mode (EFEM) derived from the even fill to construct the solution. Projecting Eq. (1) with the wake voltage in Eq. (7) to $\ell t h$ EFEM one obtains:

$$
\begin{gathered}
\ddot{\tau}^{l}+\frac{2}{\tau_{\text {rad }}} \dot{\tau}^{l}+\omega_{s}^{2} \tau^{l}=\frac{j \eta e \omega_{r f}}{E_{0} T_{0}} \sum_{m=0}^{M-1} I_{l-m} Z_{l m}(\omega) \tau^{m}, \\
I_{p}=\sum_{k=0}^{M-1} \frac{q_{k}}{T_{0}} e^{-j p k \frac{2 \pi}{M}}
\end{gathered}
$$


is the complex amplitude of the $p^{t h}$ revolution harmonic in the beam spectrum. Assume the frequency shift is small:

$$
\begin{aligned}
& \dot{\tau}^{l}+\left(\frac{1}{\tau_{\text {rad }}}-j \omega_{s}\right) \tau^{l}=\sum_{m=0}^{M-1} A_{l m} \tau^{m}, \\
& A_{l m}=\frac{\eta e f_{r f}}{2 E_{0} \nu_{s}} I_{l-m} Z_{l m}\left(\omega_{s}\right), \\
& Z_{l m}(\omega)=Z^{\| e f f}\left(l \omega_{0}+\omega\right)-Z^{\| e f f}\left[(l-m) \omega_{0}\right],
\end{aligned}
$$

where $Z^{\operatorname{leff}}(\omega)$ is defined by Eq. (6). Matrix A is the coupled matrix with dimension $M \times M$. It is constructed by the beam spectrum and the impedance. There are two terms in $Z_{l m}$ and they have different effects on the system.

\subsection{Modulating coupling}

One of the features of the uneven fill is that it introduces the mechanism of modulating coupling. If there exists one EFEM mode $\tau^{m}$ through the filling pattern it is possible to excite another EFEM mode $\tau^{l}$. Modulating coupling is represented by the term $I_{l-m} Z^{\| e f f}\left(l \omega_{0}+\omega_{s}\right)$. The idea to suppress the coupled-bunch beam instability is to design a filling pattern to couple one unstable EFEM $l$ with one stable EFEM $m$. This combination will reduce the growth rate of beam instability. The method is to design a filling pattern with the term $I_{p=l-m}$ as large as possible and the other coupling terms as small as possible. Doing this we can get an equivalent A-matrix which is diagonal except for the coupling between $\tau^{m}, \tau^{l}$. The solution of this is[1]:

$\lambda_{ \pm}=\frac{1}{2}\left(\lambda_{l}+\lambda_{m}\right) \pm \frac{1}{2} \sqrt{\left(\lambda_{l}-\lambda_{m}\right)^{2}+4 C_{l-m}^{2} \lambda_{l} \lambda_{m}}$

where $C_{p}$ is modulation parameter defined by $C_{p}=\frac{\left|I_{p}\right|}{I_{0}}$.

\subsection{Landau damping}

Another feature of uneven fill is that it introduces additional Landau damping to the system. The term Landau damping used here is different from the usual usage for the damping mechanism provided by the intrabunch frequency spread. However the concept can be adopted to explain the extra damping due to the small frequency difference of each bunch of the uneven fill. The Landau damping is provided by the inter bunch frequency spread, which is represented by the term $I_{l-m} Z^{\| \operatorname{eff}}\left[(l-m) \omega_{0}\right]$. The frequency shift of bunch $k$ relative to the mean frequency is

$$
\delta \omega_{s}^{k}=j \frac{\eta e f_{r f}}{2 E_{0} \nu_{s}} \sum_{l=1}^{M-1}\left[Z^{\| e f f}\left(l \omega_{0}\right) I_{l} e^{j 2 \pi k l / M}\right] .
$$

The Landau damped eigen value $\lambda^{L}$ is given by the dispersion relation:

$$
\frac{1}{\lambda_{0}}=\frac{1}{M} \sum_{k=0}^{M-1} \frac{1}{\lambda^{L}-j \delta \omega_{s}^{k}} .
$$

If there is an EFEM with mode index $\mathrm{n}$ in the accelerator, to fully utilize the Landau damping effect the filling pattern should maximize the beam spectrum $I_{n}$ term.

\section{NUMERICAL SIMULATION}

In the simulation we assume there are a total of $\mathrm{M}$ bunches in the accelerator. The bunches are taken to be point microparticles. The longitudinal motion of the bunches can be described by iterating a pair of two first order differential equations. The single turn map can be expressed as :

$$
\begin{aligned}
& \phi_{i, n+1}=\phi_{i, n}+2 \pi \mathrm{h} \eta \delta_{\mathrm{i}, \mathrm{n}} \\
& \delta_{i, n+1}=\delta_{i, n}\left(1-\frac{2 T_{0}}{\tau_{r a d}}\right)+ \\
& \frac{e \tilde{V}_{r f}}{E_{0}}\left(\sin \left(\phi_{i, n}+\phi_{s i, n}\right)-\sin \phi_{s i, n}-v_{i n d}\right),(17)
\end{aligned}
$$

where $\phi=\omega_{r f} \tau$ is the phase difference of the particle with the synchronous phase $\phi_{s}, \delta=\frac{\Delta E}{E_{0}}$ is the relative energy difference between the particle and the synchronous particle, $i$ labels the bunches and $n$ labels the turns, $\mathrm{h}$ is the harmonic number, $\tilde{V}_{r f}$ is the amplitude of the rf voltage, $v_{\text {ind }}$ is the voltage induced by the beam given in Eq. (7) without the parasitic loss term. Since the parasitic loss causes a constant shift of the synchronous phase it is absorbed into the synchronous phase $\phi_{s}$. For a HOM with resonance frequency $\omega_{r}$, quality factor $\mathrm{Q}$, Shunt impedance $R_{s}$, the wake function can be described as

$W_{0}^{\prime}(\tau)=\frac{\omega_{r} R_{s}}{Q} e^{\frac{\omega_{r} \tau}{2 Q}}\left(\cos \bar{\omega} \tau+\frac{\omega_{r}}{2 Q \bar{\omega}} \sin \bar{\omega} \tau\right), \quad \tau<0$

where $\bar{\omega}=\sqrt{\omega_{r}^{2}-{\frac{\omega_{r}}{2 Q}}^{2}}$. This form of wake function can be written as a phasor. The propagation of this wake function satisfies the phasor condition[2]. It simplifies the calculation of the wake function in the turn by turn tracking.

The major source of longitudinal impedance of TLS storage ring is from the Doris type rf cavities. The high-order modes of Doris cavity was numerically analyzed using a 3-D code GdfidL[3]. Our study indicates the most harmful longitudinal impedance has resonance frequency 744.1948 $\mathrm{MHz}$, loaded Q 2219 and $\frac{R_{s}}{Q}=31.95 \Omega$. The harmonic number of TLS is 200. This frequency will excite coupled bunch mode 98 . But from the observation on the real machine[3], the resonance frequency of the higher order mode is around $742 \mathrm{MHz}$. The following study will base on the same $\mathrm{Q}$ and $\frac{R_{s}}{Q}$ but shift the resonance frequency one revolution frequency down to mode number 97 .

\section{RESULTS AND DISCUSSIONS}

In the simulation the resonance frequency of worst case of the HOM is chosen, i.e. the resonance frequency rests on the first syncrotron sideband at the revolution harmonics. In this case $f_{r f}+97 f_{0}+f_{s}$ is $742.01899 \mathrm{MHz}$. We also include $5 \mathrm{~ms}$ radiation damping time in the simulation. There are in total four filling patterns used for simulation. The first one is uniform filling with total beam current 200 $\mathrm{mA}$ equally distributed in 200 bunches. The growth rate of mode 97 and damping rate of mode 103 in this case are 
calculated and shown in table 1 . The second to fourth patterns named uneven1, uneven 2 and uneven 3 are designed to utilize the coupling modulation to couple EFEM 97 and EFEM 103 to suppress the beam instability. These are the filling patterns designed to maximize the beam spectrum $I_{6}$. The filling patterns and corresponding beam spectra are shown in figure 1 . The patterns are designed in six subsets in order to maximize $I_{6}$. Pattern uneven 1 has the same current of each filled bunch. Pattern uneven 2 is same with uneven 1 except the number of filled bunch is almost half and the bunch current is doubled. Pattern uneven 3 has the same number of filled bunches as uneven 1 but the current of each subset is distributed as half sine. The total beam current of all three patterns is $200 \mathrm{~mA}$. The modulation parameter $C_{6}$ is calculated and shown in table 1 . The eigen value of the mixed modes according to Eq. (13) and corresponding Landau damping term are also calculated and shown in table 1 .
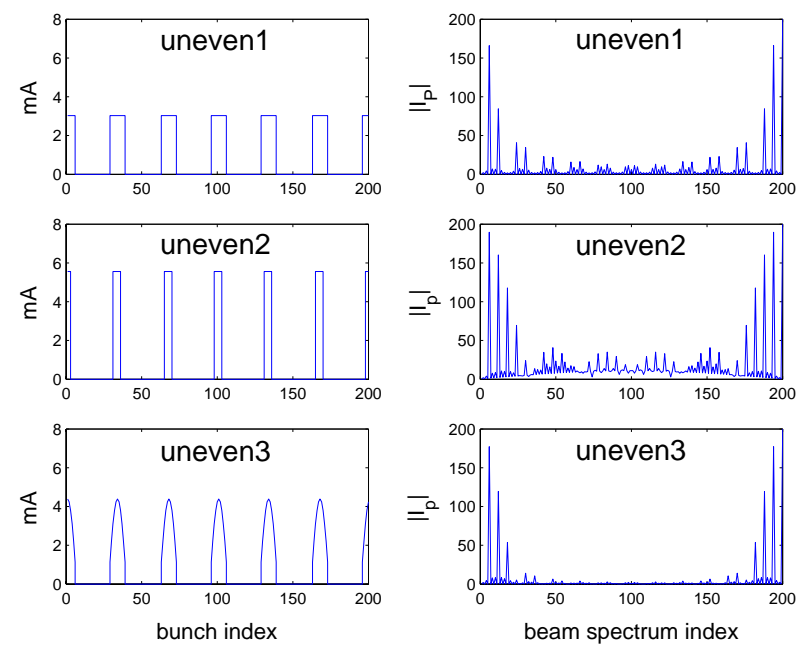

Figure 1: Filling patterns used in simulation.

Table 1: Theoretical values of modulation parameter and growth rate of uniform filling and uneven filling patterns.

\begin{tabular}{|l|c|c|c|c|}
\hline & \multirow{2}{*}{$C_{6}$} & \multicolumn{3}{|c|}{ growth rate $\mathrm{ms}^{-1}$} \\
\cline { 3 - 5 } filling pattern & $C_{6}$ & EFEM 97 & \multicolumn{2}{|c|}{ EFEM 103 } \\
\hline uniform & 0 & \multicolumn{2}{|c|}{2.1562} & \multicolumn{1}{|c|}{-1.9699} \\
\hline \hline filling pattern & $C_{6}$ & $\lambda_{+}$ & $\lambda_{-}$ & $\lambda_{\text {Landau }}$ \\
\hline uneven1 & 0.8302 & 1.2458 & -1.0595 & 2.0822 \\
uneven2 & 0.9476 & 0.7581 & -0.5718 & 1.9722 \\
uneven3 & 0.8855 & 1.0553 & -0.8698 & 2.0682 \\
\hline
\end{tabular}

The simulation results are displayed as the evolution of EFEM 97 and EFEM 103 with time in Fig. 2. The display is obtained from the turn by turn data of every bunches and project the whole information into EFEMs space. The growth rate of EFEM 97 and EFEM 103 by fitting the curve in Fig. 2 are shown in table 2. Note that the derived growth rate includes $5 \mathrm{~ms}$ radiation damping time.

From the above results the special design filling pattern does help to damp the beam instability. The simulation results are consistent with the theory. Though in the worst case the instability still grows. But in real operation the HOM can be tuned to moderate strength and by using the uneven fill the beam instability can be suppressed. In designing the special filling pattern the period of subsets is determined by the difference of mode number of two EFEMs to be coupled. In the subset with less filled bunches one derives higher modulation parameter thus lower growth rate of the mixed modes. But the current per bunch will increase and may cause single bunch beam instability. Since two modes are mixed through coupling, their growth rates should be the same. This is also demonstrated in the study. The effct of Landau damping in this study is small since the filling pattern is not designed to fully utilize this feature.

Although we have restricted ourselves to the linear regime, the simulations can be extended to exhibit instability behavior beyond the exponential growth and into nonlinear saturation. Behaviors similar to a sawtooth effect and mode frequency multiplication have been observed.
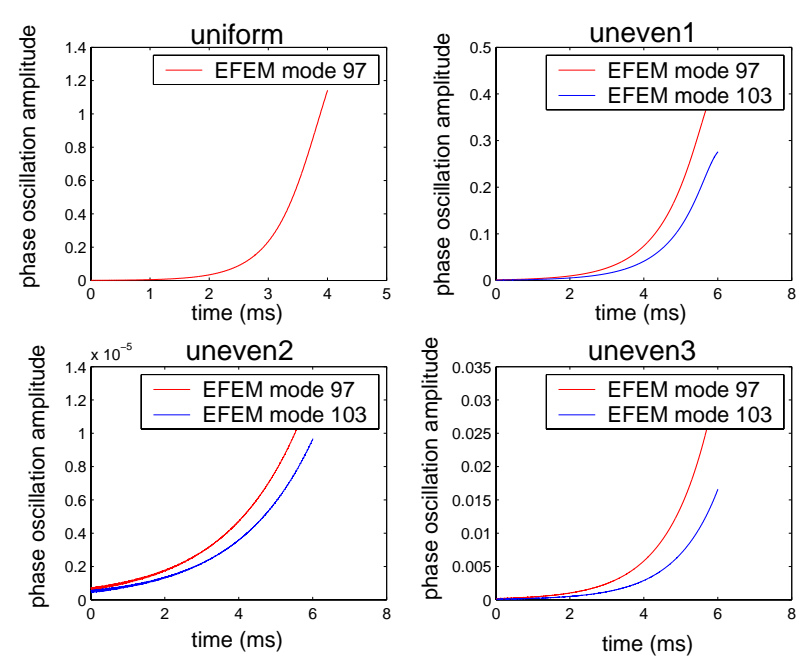

Figure 2: The evolution of EFEM 97 and EFEM 103 of four filling patterns from simulation.

Table 2: Simulation results of growth rate of EFEM 97 and EFEM 103 of the four filling patterns. Note that in the simulation $5 \mathrm{~ms}$ radiation damping time is included.

\begin{tabular}{|l|c|c|c|}
\hline & \multirow{2}{*}{ filling pattern } & $C_{6}$ & \multicolumn{2}{|c|}{ growth rate $\mathrm{ms}^{-1}$} \\
\cline { 3 - 4 } & 0 & EFEM 97 & EFEM 103 \\
\hline uniform & 0.9399 & - \\
uneven1 & 0.8302 & 1.0004 & 1.0182 \\
uneven2 & 0.9476 & 0.4947 & 0.4947 \\
uneven3 & 0.8855 & 0.8659 & 0.8703 \\
\hline
\end{tabular}

\section{REFERENCES}

[1] S. Prabhakar, Ph.D. thesis, Stanford University, SLAC-R$554,2000$.

[2] A. W. Chao, Physics of Collective Instabilities in High Energy Accelerator, Wiley 1993.

[3] P. J. Chou, A. W. Chao, "Collective beam instabilities in the Taiwan Light Source", this conference. 